Human Vision and Electronic Imaging XIV, B. E. Rogowitz and T. N. Pappas, eds.,

Proc. SPIE Vol. 7240, (San Jose, CA), Jan. 19 - 22, 2009.

\title{
Perceptual Dimensions for a Dynamic Tactile Display
}

\author{
Thrasyvoulos N. Pappas ${ }^{a}$, Vivien C. Tartter ${ }^{b}$, Andrew G. Seward ${ }^{a}$, Boris Genzer ${ }^{b}$, \\ Karen Gourgey ${ }^{c}$, and Ilona Kretzschmar ${ }^{d}$ \\ ${ }^{a}$ EECS Department, Northwestern University, Evanston, IL 60208; \\ ${ }^{b}$ Psychology Department, City College, New York, NY 10031; \\ ${ }^{c}$ Computer Center for Visually Impaired People, Baruch College, New York, NY 10010; \\ ${ }^{d}$ Department of Chemical Engineering, City College, New York, NY 10031
}

\begin{abstract}
We propose a new approach for converting graphical and pictorial information into tactile patterns that can be displayed in a static or dynamic tactile device. The key components of the proposed approach are (1) an algorithm that segments a scene into perceptually uniform segments; (2) a procedure for generating perceptually distinct tactile patterns; and (3) a mapping of the visual textures of the segments into tactile textures that convey similar concepts. We used existing digital halftoning and other techniques to generate a wide variety of tactile textures. We then conducted formal and informal subjective tests with sighted (but visually blocked) and visually-impaired subjects to determine the ability of human tactile perception to perceive differences among them. In addition to generating perceptually distinguishable tactile patterns, our goal is to identify significant dimensions of tactile texture perception, which will make it possible to map different visual attributes into independent tactile attributes. Our experimental results indicate that it is poosible to generate a number of perceptually distinguishable tactile patterns, and that different dimensions of tactile texture perception can indeed be identified.
\end{abstract}

Keywords: Tactile interface, haptic perception, tactile perception, digital halftoning

\section{INTRODUCTION}

The world is increasingly dominated by multimedia technology for communication, commerce, entertainment, art, and education. Since modern electronic media is rich in graphical and pictorial information, it has been hard for the population of visually impaired people to keep up. While some graphical and pictorial information can also be presented as speech or Braille text, the ability to present graphical and pictorial information in tactile form would dramatically increase the amount of information that can be made available to the visually impaired segment of the population. Advances in the tactile modality will also have a major impact on the development of intuitive and natural interfaces for a plethora of other applications, including virtual reality, interfaces with tactile feedback, as well as medical applications.

The tactile sense has so far received little attention, due to the lack of versatile devices. However, a number of recent advances in tactile display technology have provided impetus for new research. One of the primary motivations for our work was the development, under a current NSF project, of the "dynamic tactile tablet (DTT)," a dynamic tactile interface that is based on an electrically addressable polymeric film. In addition, a number of static tactile devices, such as Braille printers that can emboss tactile patterns on plain paper (e.g., see Ref. 1), and a "talking tactile tablet" that uses tactile patterns embossed on plastic [2], are readily available. Such devices typically offer a fully addressable array of elements that can be flat or raised at one or more heights. Some devices may also allow these elements to vibrate at different frequencies. To take advantage of the capabilities that such devices offer in order to enable the presentation of graphical, pictorial, and other information, in tactile form, it is important to understand the properties of the device and human tactile perception. The goal of this paper is to propose a new approach for converting images into tactile patterns that relies on models of the display device and human tactile and visual perception.

Further author information: Send correspondence to T. N. Pappas at email: pappas@eecs.northwestern.edu 
One of the first and most basic facts that one has to take into consideration when trying to convey visual information through the sense of touch is that human tactile perception is very different from visual perception. Loomis [3] lists several sensory and perceptual factors that differentiate the touch and visual and auditory senses, explaining the limited success of efforts at representing visual and acoustic information via the sense of touch, and why this remains a challenging problem today. The most important of these factors is limited spatial resolution. Another key consideration is haptic space perception [4].

The limited spatial resolution of the tactile sense necessitates a segmentation-based approach for transforming visual to tactile information. The idea is illustrated in Figure 1. In order to transform an array of image intensities into a pattern of bumps to be displayed on the tactile device, we first segment the image (a) into perceptually uniform - and semantically important - segments (b), then we map each segment into a distinct tactile pattern (c). Each black dot in (c) represents a bump, typically with a spherical tip of fixed radius and height. As we will see, different bump heights and vibration frequencies can also be considered. To accomplish this goal, several steps will be needed: (1) an algorithm that will segment a scene into meaningful (perceptually uniform) segments; (2) a procedure for generating perceptually distinct tactile patterns; and (3) a mapping of the visual textures of the segments into tactile textures that convey similar concepts. In many cases the visual information is already available in segmented form, for example, in maps, pie charts, and other graphics; in such cases, only steps (2) and (3) are needed. Finally, if the system is to work, the brain must be able to integrate all the information into a mental picture, which necessitates an understanding of haptic space perception.

One of the keys to achieving our goal of visual to tactile image transformation is a new approach to image segmentation and semantic classification [5-9]. We utilize the perceptual color-texture segmentation algorithm proposed in Ref. 5 that combines knowledge of human perception with an understanding of signal characteristics in order to segment natural scenes into perceptually uniform regions. It is aimed at segmentation of visual data from the real world (natural scenes), in which color and texture do not typically exhibit uniform statistical characteristics. The new approach is based on perceptual, spatially adaptive, color composition and spatial texture features that provide a simple and effective characterization of texture, which can be used to obtain robust, and at the same time, accurate and precise segmentations.

A critical component of the proposed approach is the generation of perceptually distinguishable tactile textures. As we saw above, this depends on both human tactile perception and the properties of the device. Our main focus will be on the DTT, but our results can be easily extended to other devices. We assume that the device is a fully addressable array of sites, which can be in one of two possible states: raised or flat. Eventually, it will also be possible to assume that each site can be raised to different heights and can vibrate at different frequencies. For the experiments of this paper, we have relied on existing techniques, e.g., digital halftoning, to generate a wide variety of textures. Our initial approach has been to generate a number of different tactile textures and rely on formal and informal subjective tests with sighted (but visually blocked) and visually-impaired subjects to determine the ability of human tactile perception to perceive differences among them. In addition to generating perceptually distinguishable tactile patterns, our goal is to identify significant dimensions of tactile texture perception, which will make it possible to map different visual attributes into independent tactile attributes.

The mapping from visual textures to tactile textures that convey similar concepts is not obvious because, while for some attributes of visual textures (e.g., directionality, regularity) there are straightforward tactile

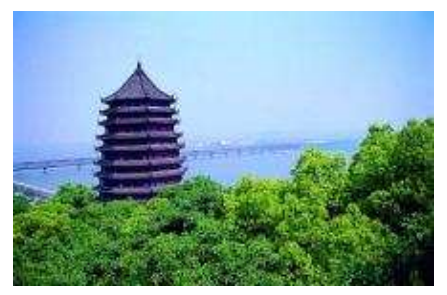

(a)

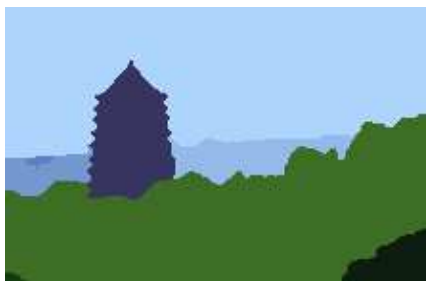

(b)

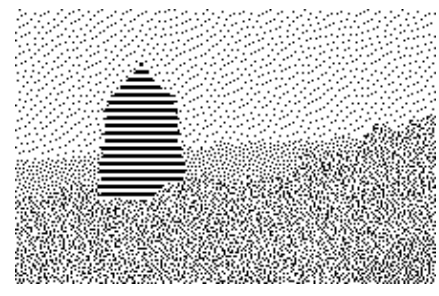

(c)

Figure 1. Visual to tactile mapping. (a) Original color image (b) Segmentation (c) Tactile display 
analogs, for others (e.g., color) there is no obvious correspondence. For such attributes it is necessary to assign an arbitrary mapping from visual concept (e.g., green grass, blue mountains) to tactile pattern. We refer to these as indirect semantic mappings in contrast to direct mappings, which are also based on semantics. Both direct and indirect semantic mappings can be used. A similar approach is used in sign language; some symbols are direct and intuitive and some are not. Direct mappings can be used when there is a direct intuitive relationship between visual and tactile features. Indirect mappings will require the extraction of semantic information, which can be done using the techniques in Refs. 8-11. While one should try to make such mappings as intuitive as possible, it is necessary to provide a mechanism for establishing the association between the visual attribute and the tactile pattern it is mapped to. For example, this can be done by training or using acoustic cues.

The final step is to verify the brain's ability to integrate all the information into a mental picture. Current and past studies have focused on the anisotropic nature of haptic space perception, for example matching the lengths of linear segments and estimates of line orientation [4]. However, the focus of our research is on scene perception, which depends on the relative location of image segments, and is quite robust to anisotropies. We believe that the presentation of a picture as a collection of segments with different tactile textures has considerable advantages over line drawings [12-15], e.g., raised lines that represent the boundaries of the segments.

\subsection{Prior Work}

A comprehensive review of the sense of touch can be found in Ref. 4. As we mentioned above, the sense of touch has not received as much attention as vision and hearing due to the lack of versatile devices. Our focus here is on surface properties of objects, and in particular the pattern-sensing capabilities of touch [3], as opposed to material properties and three-dimensional shape. While past research has examined threshold responses (e.g., two-point discrimination and temporal resolution) $[3,4]$, and texture roughness as it relates to the density of ridges and gaps of the texture gratings [16-19], there has been little work on other dimensions of tactile texture perception, e.g., regularity versus randomness [20], directionality [21], and busyness versus sparsity. Moreover, recent studies on the dimensions of haptic space have focused on real material samples [22], as opposed to surface patterns on a given material, which is the topic of our research.

Among different possible dimensions of tactile perception, there has been considerable work on texture roughness and how it relates to the density of ridges and gaps of the texture gratings [16-19]. However, most psychophysical studies have used gratings with idealized shapes, e.g., triangular and trapezoidal one-dimensional gratings [17] and conical or cut conical two-dimensional gratings [16] that are made of metal or hard plastics. On the other hand, our experiments indicate that the properties of the display (dot shape, friction) have a significant effect on texture perception.

As we saw above, one of the reasons that there has been relatively little attention devoted to touch is the lack of affordable dynamic display devices. A device that has proven to be quite effective in providing visual information and assisting visually impaired people with certain visual tasks is the tongue display [23]. It consists of an array of electrodes that can apply different voltages to stimulate the tongue, which is the most sensitive tactile organ and has the highest spatial resolution. The device relies on direct translation of grayscale video signals to voltages. Even though the size of the electrode array is limited $(12 \times 12)$, when the video camera is placed on the head, the brain makes use of superresolution principles to obtain higher resolution images. However, the aversion factor for this device is quite high. The same is true for presentation of electrical and other tactile stimuli on other parts of the body (back, abdomen, etc.). The majority of visually impaired people find such presentations quite invasive, and prefer to scan (or explore) with the finger [24]. Thus, in our research we assumed that the tactile patterns are actively scanned by the fingertip.

Another attempt at the direct rendition of grayscale values was proposed by Barner et al. [25-27], who used digital halftoning for tactile imaging. However, halftoning is not very effective when the resolution (of sensing and device) is limited, in contrast to printing. Since they are relying on a monotonic relationship between image intensities and tactile textures, the versatility of the displayed textures is quite limited. In contrast, our goal is to establish a semantic mapping between the visual and tactile textures, which allows a lot more flexibility in the choice of patterns. 


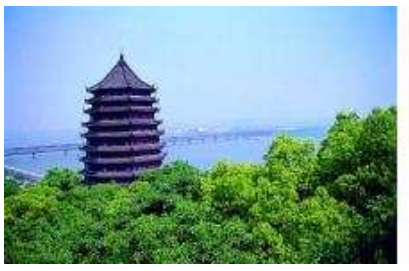

(a)

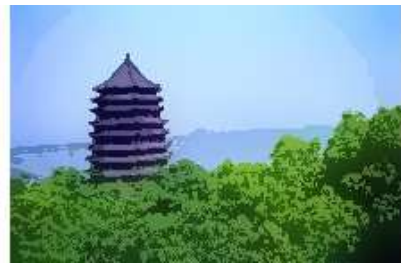

(b)

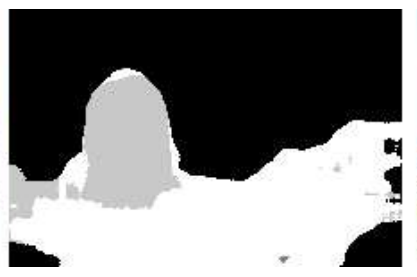

(c)

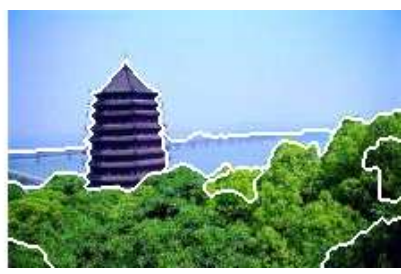

(d)

Figure 2. Color-texture features and segmentation. (a) Original image. (b) Adaptive dominant colors. (c) Texture classes (smooth regions shown in black, horizontal in gray, and complex in white). (d) Segmentation

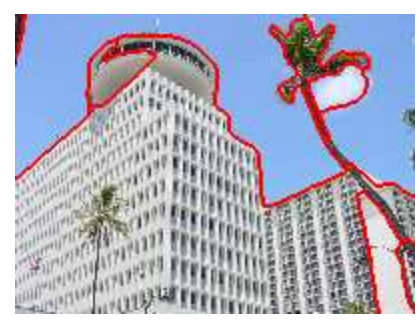

(a)

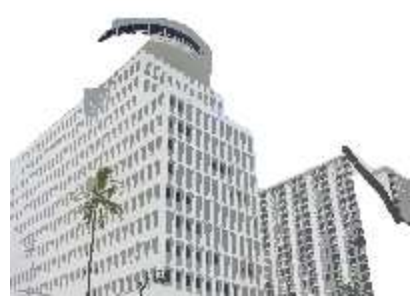

(b)

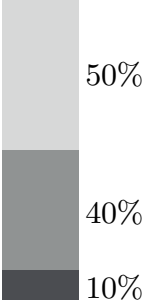

(c)

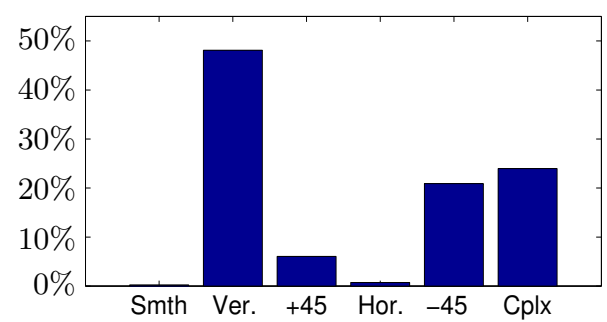

(d)

Figure 3. Segment Feature Extraction. (a) Segmented Color Image. (b) Selected Segment. (c) Its Color Composition. (d) Its Texture Composition.

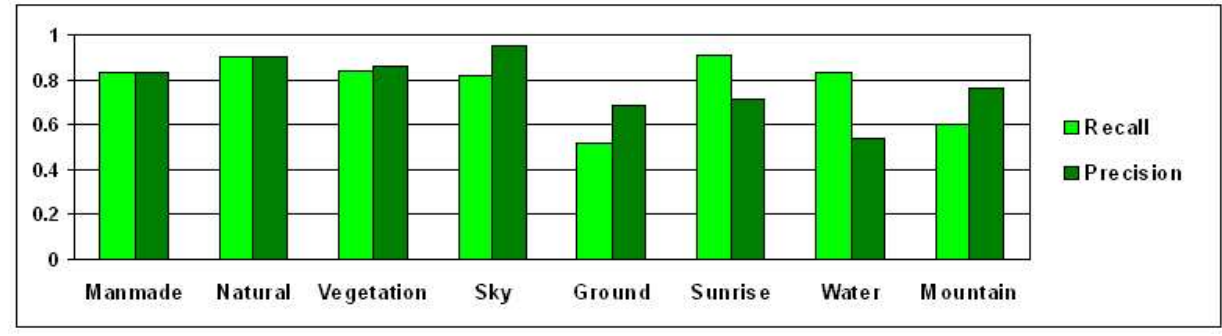

Figure 4. Classification results

The remainder of the paper is organized as follows. Section 2 reviews image segmentation and classification. Section 3 presents models for the tactile display device. In Section 4 we discuss techniques for tactile pattern generation. Section 5 presents and discusses the experimental results.

\section{REVIEW OF PERCEPTUAL IMAGE SEGMENTATION AND CLASSIFICATION}

In Ref. 5 a new image segmentation approach was proposed that is based on spatially adaptive color composition and spatial texture features. Segmentation of natural scenes is particularly difficult because, unlike artificial images that are composed of more or less pure textures, the texture characteristics of perceptually distinct regions are not statistically uniform due to effects of lighting, perspective, scale changes, etc. The human visual system (HVS) is very good at accounting for the various effects in order to segment natural scenes into perceptually uniform regions. The new approach addresses this problem by combining perceptual models and principles of texture and color processing with an understanding of image characteristics. The key elements of the segmentation approach are shown in Figure 2. It is based on two types of features, one describes the local color composition in terms of spatially adaptive dominant colors (shown in (b)), and the other the spatial texture in terms of a small number of classes, namely smooth, horizontal, vertical, $+45,-45$, and complex regions (shown in (c)). The final segmentation, superimposed on the original image is shown in (d). 
The features that form the basis for the segmentation approach can also be used to obtain segment-wide features that characterize the entire segment. Such medium-level descriptors are the key to bridging the gap between low-level image primitives and high-level semantics. An example of such descriptors is shown in Figure 3, where (a) shows a segmented image, (b) a selected segment, and (c) the color composition of the segment (dominant colors and percentages). The texture features of the segment can be similarly described by the percentage of smooth, horizontal, vertical, $+45^{\circ},-45^{\circ}$, and complex pixels as shown in Figure 3(d). In addition to color and spatial texture, the medium level descriptors may include the segment location, size, and boundary shape. The features of the segmented regions can be related to semantic labels that can then be combined to classify the image into semantic categories $[8,9]$. A variety of techniques were tried in Refs. 8,9 for segment classification, including linear discriminant analysis (LDA), Gaussian mixture models, and support vector machines. Figure 4 shows segment classification results based on a database of 3300 photographic images, based on LDA for training and classification.

As we discussed, due to limitations of the tactile sense, it is important to limit the number of segments as well as the number of semantic categories into which the segments are classified. These goals can be achieved by adjusting the parameters of the segmentation and classification algorithms.

\section{TACTILE DEVICES}

As we discussed in the introduction, a number of tactile devices, both static and dynamic, have been developed. Our primary interest is in devices that consist of a fully addressable array of elements that can be in two or more states of vertical displacement and may also be able to vibrate at different frequencies. As we saw, the existence and modeling of the tactile devices is critical for the development of techniques for visual to tactile sense substitution and an impetus for further understanding of the sense of touch.

\subsection{The "Dynamic Tactile Tablet (DTT)"}

The goal of a current NSF project (entitled "A Dynamic Tactile Interface for Visually Impaired and Blind People," Ilona Kretzschmar, PI) is to build a dynamic tactile interface that can display dynamic tactile patterns, as well as provide audio feedback on the position touched by the user. The DTT interface consists of three

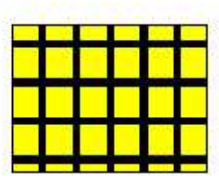

(a)

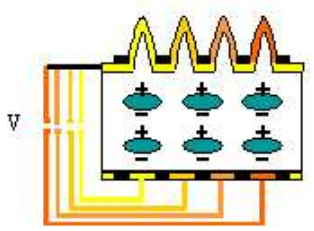

(b)

Figure 5. Schematic representation of electroactive addressable polymer film layer for dynamic tactile interface. (a) Top view and (b) Cross-sectional view.

layers. The top layer is a deformable electroactive polymer film covered with a thin film of gold; the middle layer contains electrodes that address different positions of the polymer film; and the bottom layer is a touch-sensitive screen connected to a computer to provide feedback on the position touched by the user. When a voltage is applied to the electrodes, the corresponding segment of the film extends above the film surface, creating a bump that can be sensed by the fingertip. A schematic representation of the top layer is shown in Figure 5 .

The new device will offer a number of advantages. It will have a fast dynamic response relative to the tactile sense, will be fully addressable with essentially no size limitations, and will be affordable when produced in large quantities. Another key advantage is that the polymer material of the device is pleasant to touch, which eliminates the aversion to touching the interface that many visually-impaired people have had with other devices. A prototype of the DTT device is expected to be available by the end of 2009 . 


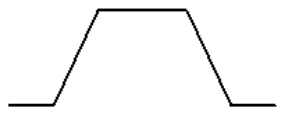

(a)

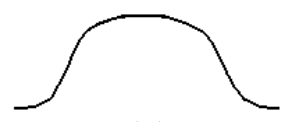

(b)

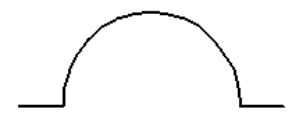

(c)

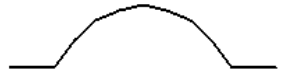

(d)

Figure 6. Device models. (a) Metal. (b) Polymer. (c,d) Casts.

\subsection{Device models}

The model of a tactile device should be based on physical considerations and other information about the device, e.g., the material and method of excitation. In this paper, we assume that the device consists of a fully addressable array of sites, which can be in one of two possible states: raised or flat. In future work we plan to consider models that allow more states, for example different bump heights and vibration frequencies. The static model applies equally well to Braille printers that emboss tactile patterns on paper [1] or plastic [2]. However, the material properties are quite different and have a significant effect on pattern perception.

In order to simulate static patterns displayed on the DTT, we used tactile test surfaces custom-made from polydimethylsiloxane (PDMS). We assume that the device has the capability to independently control the deformation at each site on a rectangular grid. The custom-made PDMS patterns allow control of the spacing, diameter, height, and shape of the dots. Physical considerations dictate that the deformations (bumps) will be circularly symmetric, bell-shaped, and at least initially, all of the same size, but may have different heights and vibrating frequencies. This is in contrast to many of the previous psychophysical experiments, which use patterns made of hard materials (metal, plastic) that have an ideal shape, such as a cut cone, a cross section of which is shown in Figure 6(a). The anticipated cross-section of the device deformations is shown in Figure 6(b). A number of other shapes have been considered by Ikei et al. [28,29]. The amplitudes of deformation for the DTT device are not yet known, but it is anticipated that they will be quantized to two or more levels. Each site will also be able to vibrate, but the achievable range of frequencies has not yet been determined. Another parameter that needs to be determined is the spacing of the dots. However, it is expected that it will be considerably shorter than that of the Braille standard [30]. In fact, one of the goals of our experiments is to determine desirable values for the device spatial resolution.

To cast the PDMS into the desired shape, we used a milling machine to create molds out of polypropylene. Examples are shown in Figure 7. To approximate the shape of the device deformations, we used hemispherical bits to mill the holes in the polypropylene molds. The cross section of the resulting bumps is shown in Figure 6(c).

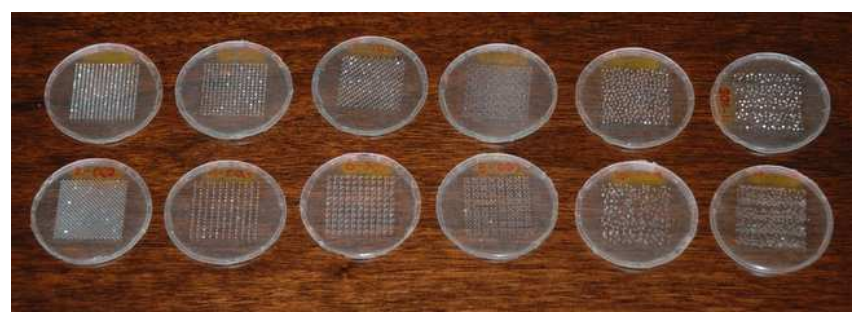

Figure 7. Tactile patterns made of cast PDMS, using polypropylene molds.

By controlling the size of the bit and the depth of the drilling, it is also possible to generate different shapes, e.g., a spherical arc with the cross-section shown in Figure 6(d). However, in the experiments described in this paper (and the samples in Figure 7), we used hemispherical dots with diameter and spacing equal to $0.8 \mathrm{~mm}$, and hence $0.4 \mathrm{~mm}$ height. The limit of tactile spatial resolution is in the $0.5-1.0 \mathrm{~mm}$ range [4]. In comparison, the Braille standard specification [30] is $1.3 \mathrm{~mm}$ diameter, $2.5 \mathrm{~mm}$ spacing, and $0.5 \mathrm{~mm}$ height. There are different versions of the Braille code that contain letters, numbers, punctuation, as well as common combinations of letters and other attributes [31]. Examples are shown in Figure 8. Individual symbols are separated by spaces. The patterns are chosen so that they are easily distinguishable and easier to read than outlines of actual letters [3]. 
Letters:

\begin{tabular}{|l|l|l|l|l|l|l|l|l|l|}
\hline $\mathrm{a}^{*}$ & $\mathrm{~b}^{:}$ & $\mathrm{c}^{*}$ & $\mathrm{~d} \because$ & $\mathrm{e} \cdot$ & $\mathrm{f}:$ & $\mathrm{g}:$ & $\mathrm{h}:$ & $\mathrm{i} \cdot$ & $\mathrm{j}:$ \\
\hline $\mathrm{k}:$ & $\mathrm{l}:$ & $\mathrm{m}:$ & $\mathrm{n}:$ & $\mathrm{o}:$ & $\mathrm{p}:$ & $\mathrm{q}:$ & $\mathrm{r}:$ & $\mathrm{s}:$ & $\mathrm{t}:$ \\
\hline $\mathrm{u}:$. & $\mathrm{v}:$ & $\mathrm{w}:$ & $\mathrm{x}:$ & $\mathrm{y}:$ & $\mathrm{z}:$ & & & & \\
\hline
\end{tabular}

Numbers:

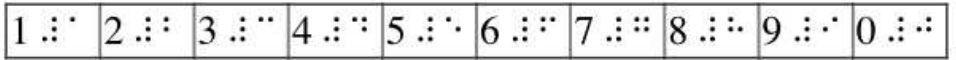

Figure 8. Braille letters and numbers.

\section{\|\|\|\|\|\|\|\|$\| \mid$}

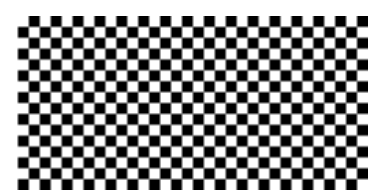

IIII!)!I!I

Figure 9. Pattern specifications (left) and realization with spacing equal to diameter (right).

However, the finger can detect the Braille patterns but not necessarily the individual dots [3]. Since our goal is to generate patterns that are perceived as a texture, the spacing and diameter of the dots should be smaller than those of Braille. In fact, if we want to have a reasonable approximation of a solid line, it may be necessary to allow some overlap between adjacent dots. This is similar to printing devices, where approximately circular dots of ink are printed on a grid, and overlap is necessary in order to be able to render solid black lines and solid black areas [32]. Figure 9 illustrates an example with no dot overlap. As we will see in the following section, there are several analogies with printing, but also, significant differences.

As we saw above, previous psychophysical studies have used gratings with idealized shapes made of metal or hard plastics. In addition to the shape difference of the deformations of the DTT device and its models, both the polymeric film of the DTT device and the static PDMS patterns are considerably softer and have more friction. This has had a significant effect in our experimental results. In future work, we plan to experiment with tactile patterns embossed on paper, which provides yet another type of material, with different microtexture (i.e., the texture of the material itself) and friction properties than PDMS or metal and hard plastics, and is thus expected to further highlight the influence of the device on the overall perceptual effect.

\section{GENERATION OF TACTILE PATTERNS}

In this section we discuss the generation of tactile texture patterns. Our goal is to generate a number of perceptually distinct tactile textures. Since we have not yet developed a good model of tactile pattern perception, our initial approach was to generate a number of different tactile textures and to rely on subjective tests with sighted (but visually blocked) and visually-impaired subjects to determine the ability of human tactile perception to perceive differences among them.

We used a variety of techniques to generate tactile textures. In addition to a number of periodic patterns, one approach for generating a wide variety of tactile patterns is through the use of digital image halftoning techniques. There is a rich literature on halftoning techniques [33-35] that depend on different principles and can generate a wide variety of binary textures, which can be used as a pattern of displacements. In the following discussion, we will focus on bilevel techniques, but those can be easily extended to multiple levels that correspond to multiple displacements or vibration frequencies. Figure 10 shows periodic patterns on the left and patterns generated by digital halftoning on the right. The patterns in (d) were generated with "Bayer" screening, the patterns in (e) with Floyd-Steinberg (FS) error diffusion (ED), without and with weight perturbations, at the top and bottom, respectively, and the patterns in (f) with FS ED applied to images with vertical ripples. Note that in this section we will show the ideal halftone patterns (black and white square pixels) as opposed to the actual patterns that the device can generate. 


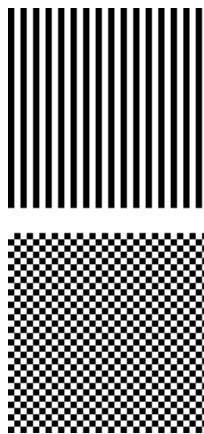

(a)

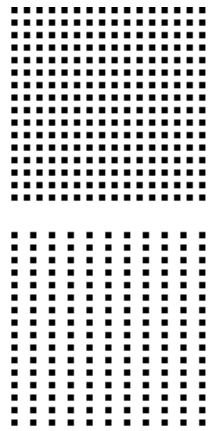

(b)

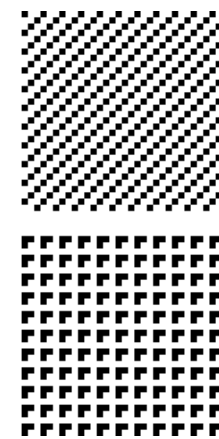

(c)

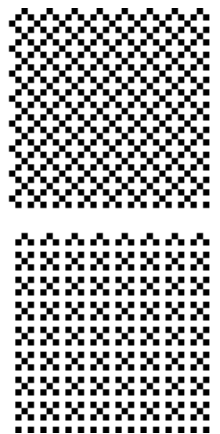

$(\mathrm{d})$

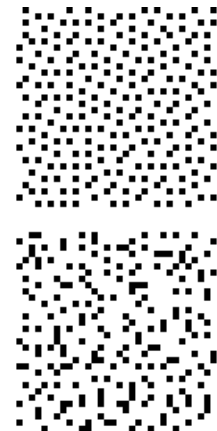

(e)

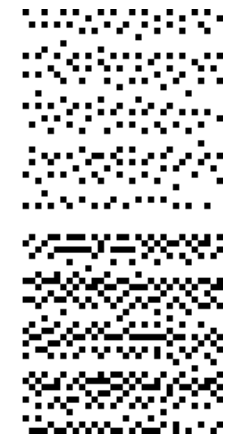

(f)

Figure 10. Tactile textures (a-c) periodic patterns (d) Bayer (e-top) error diffusion (e-bottom) error diffusion with perturbed weights (f) error diffusion of non-constant patches.

The problems of digital halftoning and tactile pattern generation appear to be quite similar. However, there is an important difference. In image halftoning, texture is used to render different tone levels, and thus, the visibility of halftone-induced textures is undesirable and should be minimized, while in the tactile case, the goal is the textures themselves, that is, we want to accentuate the characteristics of the textures we generate. For example, Figure 10(e) shows visually pleasing blue noise (i.e., at high frequencies where it is not visible) at the top, while at the bottom we have a pattern that contains more low frequencies and is considerably less pleasing. On the other hand, when blind subjects scanned these patterns with the finger, they found the pattern at the bottom "interesting" compared to the other patterns in the set, which are more regular. Thus, pattern regularity is an important discriminating feature.

As we mentioned above, Barner et al. [25-27] have used digital halftoning for tactile imaging. They have considered a variety of models (deterministic and stochastic) for generating patterns [27], and have proposed techniques that are optimized for models of the display device and human tactile sensitivity [26]. The latter extends the idea of model-based halftoning $[32,36-38]$ to the tactile domain. However, we would also like to emphasize a significant difference between the work of Barner et al. and the proposed approach for transforming visual information to tactile form. Barner et al. aim at a monotonic relationship between image intensities and tactile pattern attributes. Due to the bandwidth limitations of touch, they point out the need for processing for edge enhancement, and have also considered explicit segmentation [25], but all this in order to reduce the number of levels that they need to depict and to accentuate the region boundaries. Their goal remains to preserve a monotonic relationship between image tone levels and tactile textures. Thus, they use one halftoning technique to map the entire image. In contrast, our goal is to establish a semantic mapping between the visual and tactile textures. This can be based on segment classification or on direct mapping of the segment's visual texture attributes (color, roughness, directionality, etc.) to tactile texture attributes (roughness, vibration frequency, directionality, etc.) Thus, the tactile textures for a given image do not have to be generated by the same technique. As we pointed out in the introduction, this visual to tactile mapping is not straightforward because, for some attributes of visual textures (e.g., color), there is no obvious corresponding tactile texture. Also, some obvious mappings (e.g., based on dot density) correspond to different attributes in the two domains (tone level and roughness, respectively), and as such may not be the best for associating semantics.

Our goal is to generate tactile textures that are easily distinguishable when scanned by the finger. We also want to generate halftones along separate perceptual dimensions (e.g., directionality, roughness), which will make it easier to assign a combination of visual attributes to a particular texture. Figure 11 shows patterns generated by different halftoning techniques. The pattern density varies in the vertical direction. The first four columns are all ED; standard FS in (a), FS with weight perturbations in (b), and Jarvis-Judice-Ninke ED with different weight perturbations in (c) and (d). The fifth column is halftoning with a special type of screen, which we call "bad pebble," while the last column is random dither. Note that there is quite a variety of textures. In future research, we hope to develop metrics that can predict the perceived differences between tactile patterns, and based on those metrics to propose new pattern generation techniques that can automatically generate perceptually distinct 

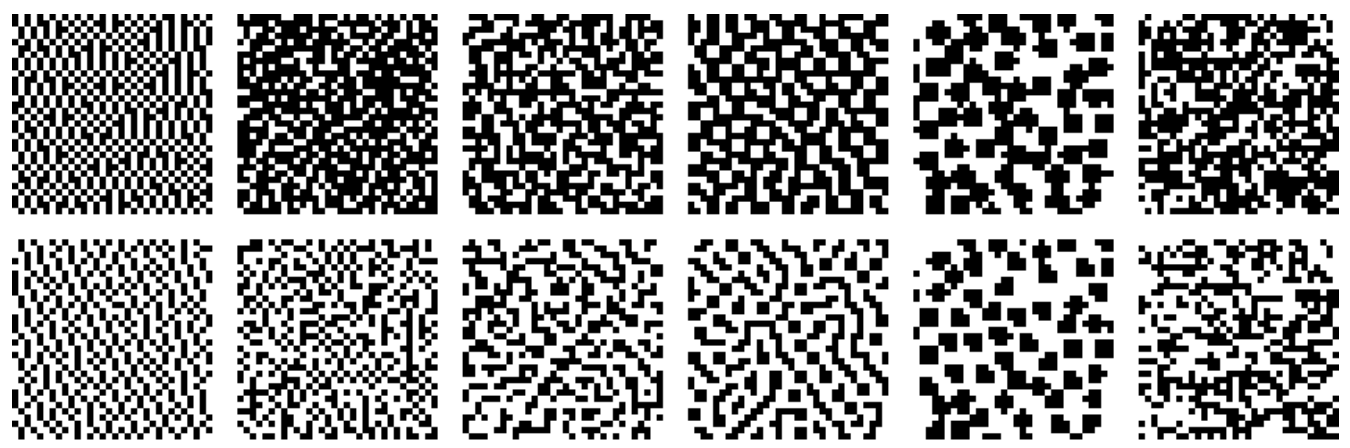

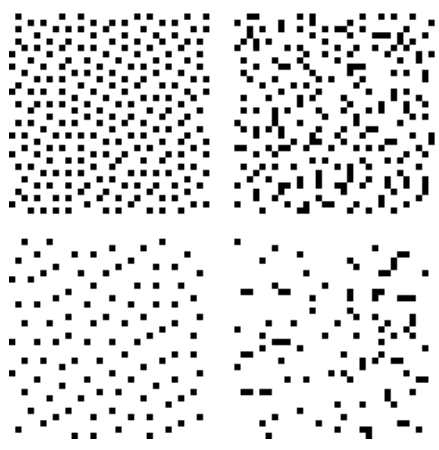

(a) (b)

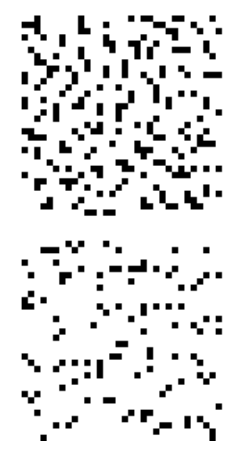

(c)

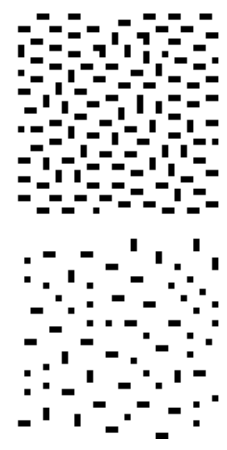

(d)

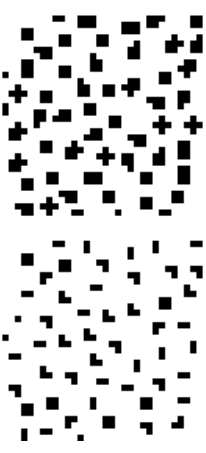

(e)

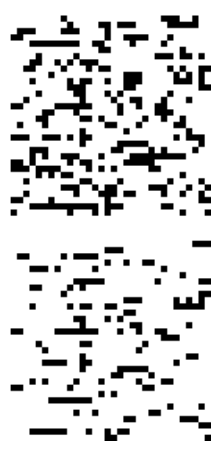

(f)

Figure 11. Tactile textures (a) error diffusion (b-d) error diffusion with different weight perturbations (e) screening with "badpebble" (f) random

tactile patterns.

\section{EXPERIMENTAL RESULTS}

We conducted two sets of subjective experiments with both (visually blocked) sighted and visually-impaired subjects. The goals of the subjective experiments were (1) to determine the ability of subjects to perceive differences between pairs of texture patterns, and (2) to determine their ability to perceive differences in specific tactile texture attributes, like roughness, regularity, directionality, etc.

In the first experiment, we used PDMS impressions of the 12 patterns shown in Figure 10. As described in Section 3, we used a milling machine to create molds out of polypropylene, then poured PDMS into the molds. The resulting samples are shown in Figure 7. The samples were circular with diameter approximately equal to 1.7 inches. The dimensions of the tactile pattern, which was centered on the disk, were $1 \times 1$ inch.

We conducted informal subjective tests with visually blocked sighted subjects and a visually-impaired subject. Based on the results, which are described below, we did not see the need for more formal tests with additional subjects. In the following we describe the results with the visually-impaired subject.

First, the stimuli were described as plastic circles with a pattern in the center. A piece of plastic that was defective was presented for the general feel. Then one of the stimuli was presented. The subject immediately found the rectangle of the pattern in the circle and it was explained that they would all be rectangles and that the task was to describe and maybe differentiate the composition of the rectangles. Each stimulus was stroked, mostly with the index and middle finger tips, up and down, and left and right. After several such strokings, the subject described her impressions. Frequently the impression was qualitative, e.g., like, dislike, or boring, followed by a more detailed description of what was sensed. In no case was there instantaneous recognition. After more than an hour the subject was tired, but with her consent, continued the experiment.

The stimulus perimeters were more critical to the subject's description detail than the patterns themselves. The "fine structure" of the patterns was not really critical: patterns with isolated dots were no different from 

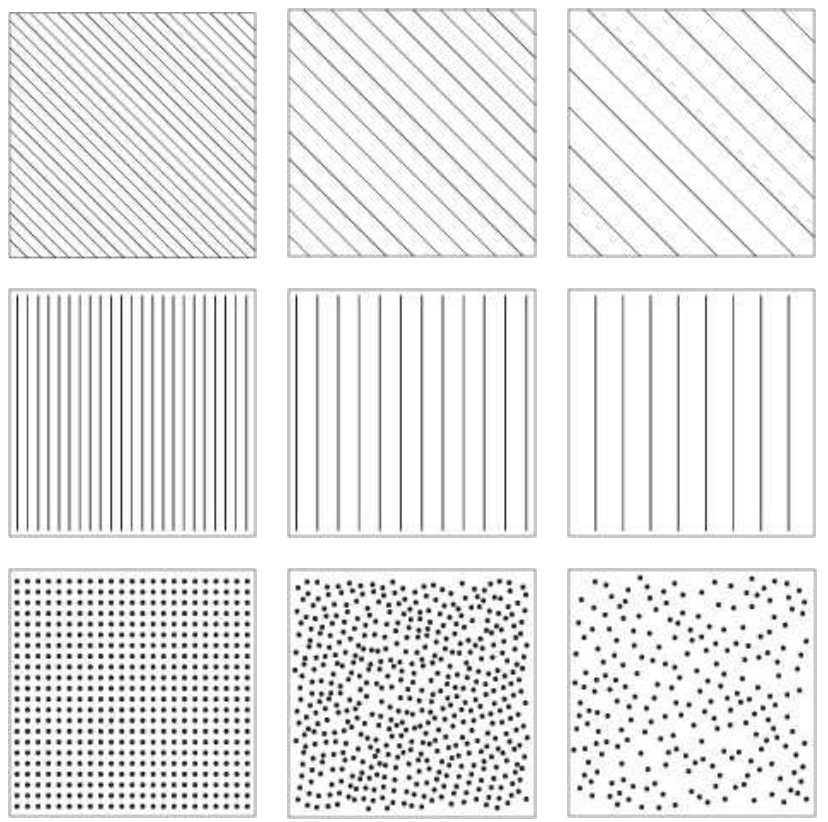
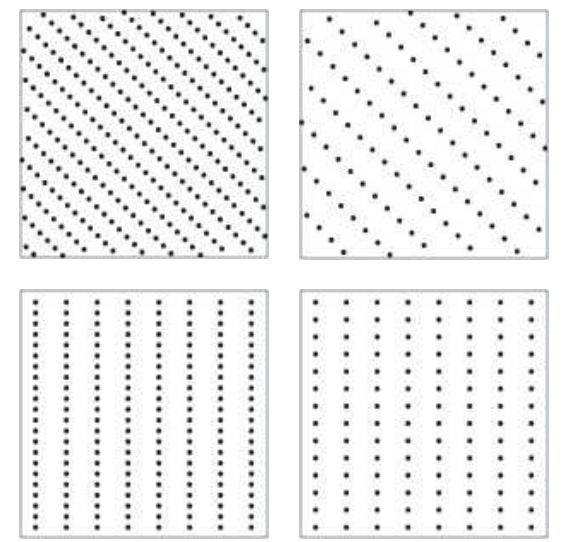

Figure 12. Tactile patterns traced with Braille stylus. Top row: diagonal solid and dotted lines with different line and dot densities. Middle row: vertical solid and dotted lines with different line and dot densities. Bottom row, left to right: uniform high-density dot pattern; high-density blue-noise pattern, low-density blue-noise pattern.

patterns that contained crosses or triangles formed by clusters of dots. Stroking of the stimuli dislodged some of the nodes, which necessitates that we improve the casting procedure to make them more durable. The subject was very sensitive to stimulus "defects," e.g., blank spaces in the pattern due to milling imperfections, which points to the sensitivity to irregular patterns. (See discussion on random patterns below.) The subject also experienced a different feel for some stimuli depending on the direction of the stroke, left versus right or up versus down. We do not have an explanation for this.

When the subject had settled with a stimulus description, a new stimulus was presented for comparison. She was first asked to describe it, then to say if it belonged in the same group as the previous stimulus or stimuli. If there were more than one stimulus groups already, she was asked which it fit better with and why. Sometimes she was asked if two stimuli were discernibly different. Sometimes three stimuli were presented, after she had grouped them, and she was asked to select the oddball, the one that did not belong. Overall, the testing was not as systematic as we might have wished, but it was clear that little changed on second impressions.

Without going into a detailed description of subject's response to the different stimuli, the general conclusion was that it was very difficult to discriminate between the different patterns, with one notable exception: The subject preferred asymmetric, random patterns, e.g., the bottom pattern in Figure 10(e), which she described as "interesting," compared to the symmetric, more regular patterns in Figure 10(a-d), as well as the top pattern in Figure 10(e), which we would characterize as semi-random. The results of our experiments with sighted but visually blocked subjects were similar. The conclusion is that the dot diameter and spacing were too small to reveal differences in the given pattern variations.

We then conducted a second experiment using tactile patterns imprinted on polyimide film, attached to $1.75 \times 1.75$ inch squares cut from manilla folders, using a standard Braille stylus. The patterns are shown in Figure 12. Note that since the dots and lines were traced by hand, the lines were continuous. Also, the dot spacing of the vertical and diagonal dotted lines was the same, i.e., they do not correspond to what would be displayed in a Cartesian array. The dot diameter and line width was approximately $1 \mathrm{~mm}$, and the minimum dot and line spacing was $2 \mathrm{~mm}$. More details can be found in Ref. 39 .

These stimuli were selected to explore whether subjects could differentiate between continuous and dotted lines, different line densities, different dot spacing in dotted lines, ordered and semi-random (blue-noise) dot 
patterns, vertical and diagonal lines. Sixteen sighted but visually blocked subjects and twelve visually impaired subjects participated in the experiments. The subjects were instructed to examine the patterns with the index finger.

The experiment consisted of three parts. In the first part, the subjects were asked to describe the difference between one of the stimuli and a flat anchor. As expected, the flat and textured stimuli were clearly differentiable. In the second part, the subjects were asked specific questions about the stimuli: (1) pleasant or unpleasant, (2) rough or smooth, (3) busy or sparse, and (4) random or ordered. For the stimuli that were ordered, the subjects were also asked whether there were any lines, and if they were vertical or diagonal and smooth or dotted. The third pass consisted of two-way comparisons.

The detailed results of the experiments are described in Ref. 39. Overall, the subjects were able to discriminate between the two orientations, solid and dotted lines, ordered and semi-random dot patterns, and were able to detect at least two dot or line densities. There were small differences between the sighted and visually impaired subjects, but overall the responses of the two groups were consistent.

Based on the two experiments, we can conclude that it is possible to generate a number of perceptually distinguishable tactile patterns, and that different dimensions of tactile texture perception can indeed be identified. We can also conclude that a dot diameter of $1.0 \mathrm{~mm}$ is a reasonable choice for a tactile device. More experiments will be required to determine the dot spacing, for example, to find the minimum dot spacing for which a dotted line can be differentiated from a solid line.

\section{ACKNOWLEDGMENTS}

This material is based upon work supported by the National Science Foundation under Grant No. CBET-0651747. Any opinions, findings and conclusions or recommendations expressed in this material are those of the authors and do not necessarily reflect the views of the National Science Foundation (NSF). The authors wish to thank

Agatha Cadette and Chok Yew Keong who prepared PDMS masks and ran tests at the Blind Youth Slam organized by JHU in the summer 2007.

\section{REFERENCES}

1. "ViewPlus Braille embossers." http://www.viewplus.com.

2. "Talking tactile tablet." http://www.touchgraphics.com/.

3. J. M. Loomis, "Tactile pattern perception," Perception 10, pp. 5-27, Feb. 1981.

4. R. L. Klatzky, S. J. Lederman, "Touch," in Handbook of Psychology, Volume 4: Experimental Psychology, A. F. Healy and R. W. Proctor, eds., ch. 6, John Wiley and Sons, second ed., 2003.

5. J. Chen, T. N. Pappas, A. Mojsilovic, B. E. Rogowitz, "Adaptive perceptual color-texture image segmentation," IEEE Trans. Image Processing 14, pp. 1524-1536, Oct. 2005.

6. D. Depalov, T. N. Pappas, D. Li, B. Gandhi, "A perceptual approach for semantic image retrieval," Proc. Int. Conf. Acoustics, Speech, and Signal Processing (ICASSP), II, pp. 417-420, (Toulouse, France), May 2006.

7. D. Depalov, T. N. Pappas, D. Li, B. Gandhi, "Perceptually based techniques for semantic image classification and retrieval," Human Vision and Electronic Imaging XI, B. E. Rogowitz, T. N. Pappas, and S. J. Daly, eds., Proc. SPIE Vol. 6057, pp. 6057OZ-1-6057OZ-10, (San Jose, CA), Jan. 2006.

8. T. N. Pappas, J. Chen, D. Depalov, "Perceptually based techniques for image segmentation and semantic classification," IEEE Commun. Mag. 45, pp. 44-51, Jan. 2007.

9. D. Depalov and T. N. Pappas, "Analysis of segment statistics for semantic classification of natural images," Human Vision and Electronic Imaging XII, B. E. Rogowitz, T. N. Pappas, and S. J. Daly, eds., Proc. SPIE Vol. 6492, pp. 6492OD-1-6492OD-11, (San Jose, CA), Jan. 29 - Feb. 12007.

10. T. N. Pappas, J. Chen, D. Depalov, "Learning perception," OE Magazine 5, pp. 18-20, Oct. 2005.

11. D. Depalov, Perceptual Color and Texture Features for Image Categorization and Retrieval. PhD thesis, Northwestern Univ., Evanston, IL, 2007.

12. M. W. A. Wijntjes, A. M. L. Kappers, "Angle discrimination in raised line drawings," Perception 36, pp. 865-879, 2007. 
13. M. W. A. Wijntjes, T. van Lienen, I. M. Verstijnen, A. M. L. Kappers, "Look what I have felt: Unidentified haptic line drawings are identified after sketching," Acta Psychologica 128, pp. 255-263, June 2008.

14. M. W. A. Wijntjes, T. van Lienen, I. M. Verstijnen, A. M. L. Kappers, "The influence of picture size on recognition and exploratory behaviour in raised-line drawings," Perception 37, pp. 602-614, 2008.

15. M. W. A. Wijntjes, Haptic Perception of Sahpes and Line Drawings. PhD thesis, Utrecht University, 2008.

16. C. E. Connor and K. O. Johnson, "Neural coding of tactile texture: Comparison of spatial and temporal mechanisms for roughness perception," Journal of Neuroscience 12(9), pp. 3414-3426, 1992.

17. T. Yoshioka, B. Gibb, A. K. Dorsch, S. S. Hsiao, K. O. Johnson, "Neural coding mechanisms underlying perceived roughness of finely textured surfaces," Journal of Neuroscience 21(17), pp. 6905-6916, 2001.

18. C. J. Cascio, K. Sathian, "Temporal cues contribute to tactile perception of roughness," Journal of Neuroscience 21(18), pp. 7416-7427, 2001.

19. E. Gamzu, E. Ahissar, "Importance of temporal cues for tactile spatial-frequency discrimination," Journal of Neuroscience 21(14), pp. 5289-5296, 2001.

20. B. Unger, R. Hollis, R. Klatzky, "JND analysis of texture roughness perception using a magnetic levitation haptic device," Proc. Second Joint EuroHaptics Conf. and Symposium on Haptic Interfaces for Virtual Environment and Teleoperator Systems (WHC'07), Mar. 2007.

21. B. Hughes, "Haptic exploration and the perception of texture orientations," Haptics-e 4(2), 2006.

22. W. M. B. Tiest, A. M. L. Kappers, "Analysis of haptic perception of materials by multidimensional scaling and physical measurements of roughness and compressibility," Acta Psychologica 121, pp. 1-20, 2006.

23. P. Bach-y-Rita, K. A. Kaczmarek, "Tongue placed tactile output device." US Patent \# 6430450, Aug. 2002.

24. K. Gourgey, "Personal communication."

25. S. E. Hernandez, K. E. Barner, "Tactile imaging using watershed-based image segmentation," ASSETS'00, pp. 26-33, (Arlington, VA), Nov. 2000.

26. A. Nayak, K. E. Barner, "Optimal halftoning for tactile imaging," IEEE Trans. Neural Syst. Rehabil. Eng. 12, pp. 216-227, June 2004.

27. T. C. Aysal and K. E. Barner, "Stochastic and deterministic models for haptic pseudo-textures," Haptic Interfaces for Virtual Environment and Teleoperator Systems (HAPTCS'06), pp. 469-476, (Alexandria, VA), Mar. 2006.

28. Y. Ikei, K. Wakamatsu, S. Fukuda, "Image data transformation for tactile texture display," Proc. Virtual Reality Annual Int. Symposium, pp. 51-58, IEEE, 1998.

29. Y. Ikei, M. Yamada, S. Fukuda, "A new design of haptic texture display - texture display 2 - and its preliminary evaluation," Proc. Virtual Reality, pp. 21-28, IEEE, Mar. 2001.

30. "Specification \#800, national library service, national library of congress."

31. "Braille alphabet." http://libbraille.org/alphabet.php.

32. T. N. Pappas, D. L. Neuhoff, "Printer models and error diffusion," IEEE Trans. Image Processing 4, pp. 6680, Jan. 1995.

33. R. Ulichney, Digital Halftoning, The MIT Press, Cambridge, MA, 1987.

34. T. N. Pappas, J. P. Allebach, D. L. Neuhoff, "Model-based digital halftoning," IEEE Signal Processing Mag. 20, pp. 14-27, July 2003.

35. D. L. Lau, R. Ulichney, G. R. Arce, "Blue- and green-noise halftoning models," IEEE Signal Processing Mag. 20, pp. 28-38, July 2003.

36. T. N. Pappas, D. L. Neuhoff, "Model-based halftoning," Human Vision, Visual Proc., and Digital Display II, B. E. Rogowitz, M. H. Brill, and J. P. Allebach, eds., Proc. SPIE, Vol. 1453, pp. 244-255, (San Jose, CA), Feb. 1991.

37. T. N. Pappas, D. L. Neuhoff, "Least-squares model-based halftoning," Human Vision, Visual Proc., and Digital Display III, B. E. Rogowitz, ed., Proc. SPIE, Vol. 1666, pp. 165-176, (San Jose, CA), Feb. 1992.

38. T. N. Pappas, D. L. Neuhoff, "Least-squares model-based halftoning," IEEE Trans. Image Processing 8, pp. 1102-1116, Aug. 1999.

39. V. C. Tartter, B. Genzer, T. N. Pappas, K. Gourgey, I. Kretzschmar, "Haptic perception: Immediate recognition of surface dimensions in imprinted plastic film," Perception 83 Psychophysics . Submitted for publication. 